\title{
Transtendinous course of the infrapatellar branch of saphenous nerve. A contribution to the aetiology of entrapment neuropathy and modification of the existing classification
}

\author{
K. Natsis ${ }^{1}$, G. Konstantinidis ${ }^{1}$, G. Geropoulos ${ }^{1}$, T. Totlis ${ }^{1}$, N. Lazaridis ${ }^{1}$, T. Tegos ${ }^{2}$ \\ ${ }^{1}$ Laboratory of Anatomy, Faculty of Medicine, School of Health Sciences, Aristotle University of Thessaloniki, \\ Macedonia, Greece

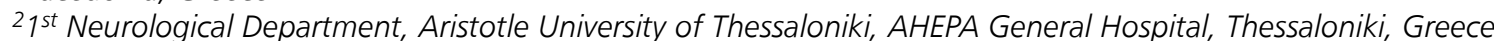

[Received: 8 February 2016; Accepted: 19 March 2016]

Background: The course of the infrapatellar branch of saphenous nerve (IPBSN) in relation to the Sartorius muscle has been classified into presartorial, transsartorial and retrosartorial types. Mechanical compression of the IPBSN within the Sartorius tendon has been surgically recognised as a cause of entrapment neuropathy. Purpose of the present study was to differentiate the IPBSNs penetrating the Sartorius tendon from those penetrating the Sartorius muscle, from an anatomical and clinical point of views and thus modifying the existing classification.

Materials and methods: The IPBSN was bilaterally dissected in 27 cadavers. The cases of the IPBSNs penetrating the Sartorius tendon were recorded separately from those penetrating the Sartorius muscle belly.

Results: In 11 out of 54 limbs (20.4\%) the IPBSN ran through the Sartorius muscle belly. In 3 out of 54 (5.6\%) limbs, the IPBSN penetrated the Sartorius tendon.

Conclusions: The penetrating type of IPBSN includes two distinct subtypes: the muscle-penetrating type and the tendon-penetrating type. These subtypes are also distinct from a clinical point of view, since only the tendon-penetrating type has been associated with the IPBSN entrapment neuropathy. According to these findings we suggest a modification of the current classification. Further clinical studies are necessary to fully demonstrate whether the tendon-penetrating type should be considered as a predisposing factor for the IPBSN entrapment neuropathy. Distinguishing the two subtypes might be helpful for that purpose. (Folia Morphol 2016; 75, 4: 481-485)

Key words: knee surgery, knee anatomy, complications, nerve compression, infrapatellar branch

\section{INTRODUCTION}

The saphenous nerve is the largest and longest sensory branch of femoral nerve. During its course along the medial aspect of the leg, it gives off a branch at the level of the knee joint, termed the infrapatellar branch of saphenous nerve (IPBSN). The IPBSN curves around the posterior border of Sartorius muscle, penetrates the fascia lata and becomes subcutaneous to supply the skin of the anterior and medial aspect of the knee. In $75 \%$ of the population

Address for correspondence: K. Natsis, MD, PhD, BSc, Professor of Anatomy, Orthopaedic Surgeon, Head of the Laboratory of Anatomy, School of Medicine, Faculty of Health Sciences, Aristotle University of Thessaloniki, P.O. Box 300, Thessaloniki, 54124, Greece, tel: +30 2310999681 , e-mail: natsis@auth.gr 


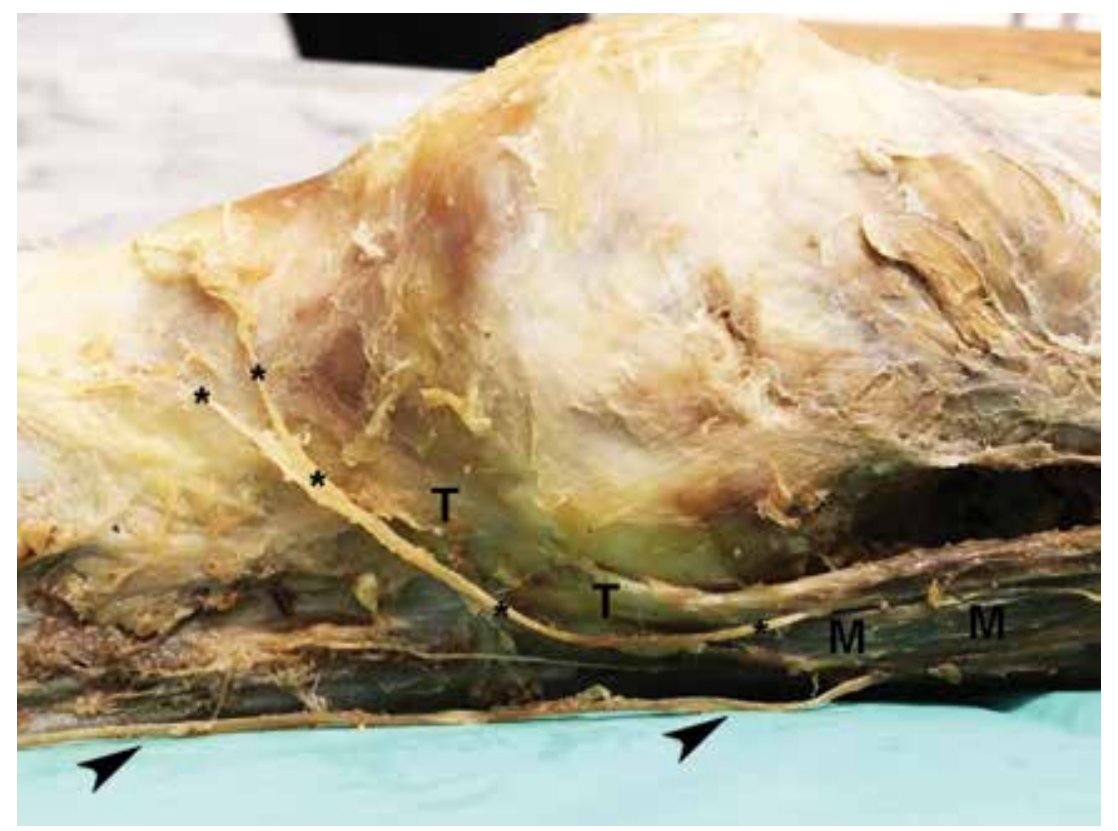

Figure 1. Infrapatellar branch $\left({ }^{*}\right)$ of the saphenous nerve (arrow heads) passing through sartorius muscle $(\mathrm{M})$ in the right limb of a male cadaver; $\mathrm{T}$ - Sartorius tendon.

the IPBSN consists of more than one branch and contributes to the formation of the peripatellar nerve plexus along with the anterior and lateral femoral cutaneous nerves. Following the formation of the IPBSN, the saphenous nerve descends to the medial side of the knee giving off cutaneous branches which supply the medial border of the tibia down to the medial side of the foot [14]. The IPBSN may consist of one to three branches [4].

In the literature, special attention has been paid to the topographic anatomy of the IPBSN since iatrogenic injury of the nerve has been reported as a complication of both knee arthroscopy and knee open surgery [3]. Moreover, other studies focused on the anatomical variations in the relationship between the IPBSN and the Sartorius muscle $[4,11]$, leading to lesions that might have clinical importance expressed as entrapment neuropathy $[8,16]$. Kalthur et al. (2015) [9] studied the course of the IPBSN in relation to the Sartorius muscle in 32 male cadavers and classified the variations into three types; anterior to Sartorius (presartorial) (68.7\%), penetrating the Sartorius (transsartorial) (28.1\%), and posterior to Sartorius (retrosartorial) (3.1\%). However, during our routine dissections we noticed that the penetrating type of the IPBSN consists of two separate subtypes; the first one passing through the muscle belly and the other piercing the tendon. Mechanical compression of the IPBSN within the Sartorius tendon has been surgically recognised as a cause of entrapment neuropathy [16]. These observations led us to conduct a study in order to differentiate the IPBSNs penetrating Sartorius tendon from those penetrating Sartorius muscle, from both an anatomical and a clinical point of view, with the aim to modify the current classification.

\section{MATERIALS AND METHODS}

The sample of the study consisted of 27 formalinembalmed (12 male and 15 female) Greek cadavers aged 58-91 years which were routinely dissected in our Laboratory. The femoral nerve was dissected in the femoral triangle in a total of 54 limbs. In addition, all the branches of the femoral nerve were dissected in the thigh. Along its course lateral and deep to the femoral vessels, the femoral nerve gave off the saphenous nerve. The roof of the sub-sartorial or Hunter's canal was opened and the saphenous nerve was followed until its division into the IPBSN and the descending branch. The IPBSN ran anteromedially towards the subcutaneous fat between the patella apex and the tibial tubercle. The cases in which the IPBSN penetrated either the Sartorius muscle belly or its tendon were recorded. The study was approved by the institutional review board.

\section{RESULTS}

In 11 out of 54 limbs (20.4\%), the IPBSN passed through the belly of the Sartorius muscle (Figs. 1, 2). 


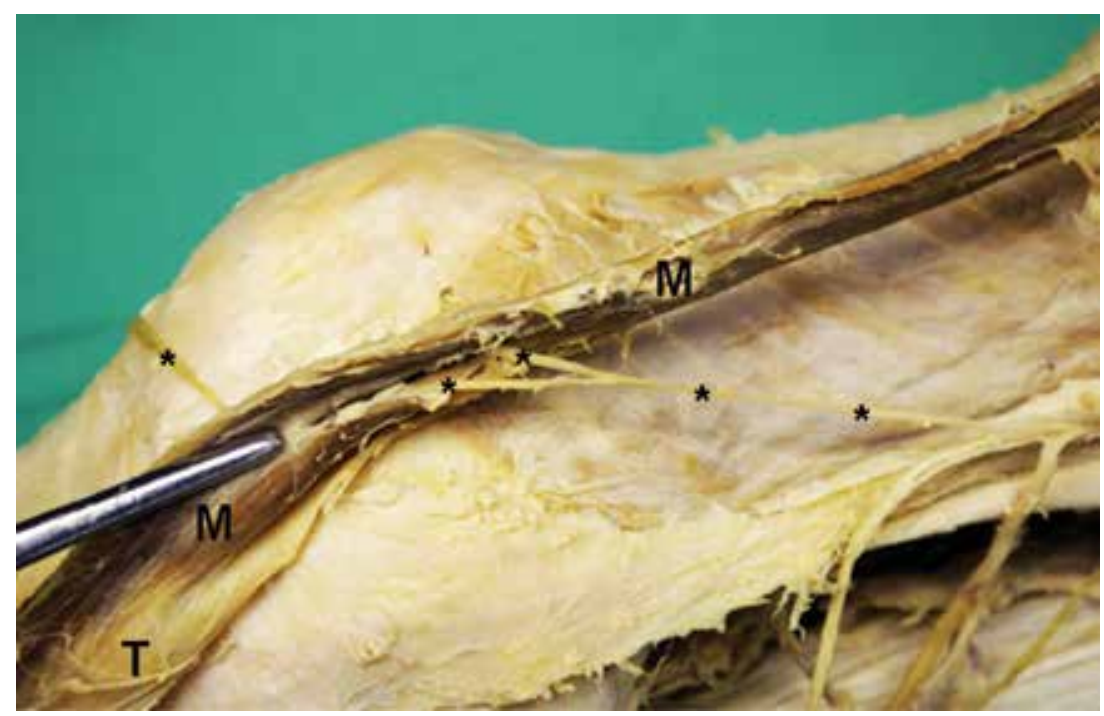

Figure 2. Two infrapatellar branches $\left(^{*}\right)$ of the saphenous nerve passing through sartorius muscle $(\mathrm{M})$ in the right limb of a male cadaver; $\mathrm{T}$ - Sartorius tendon.

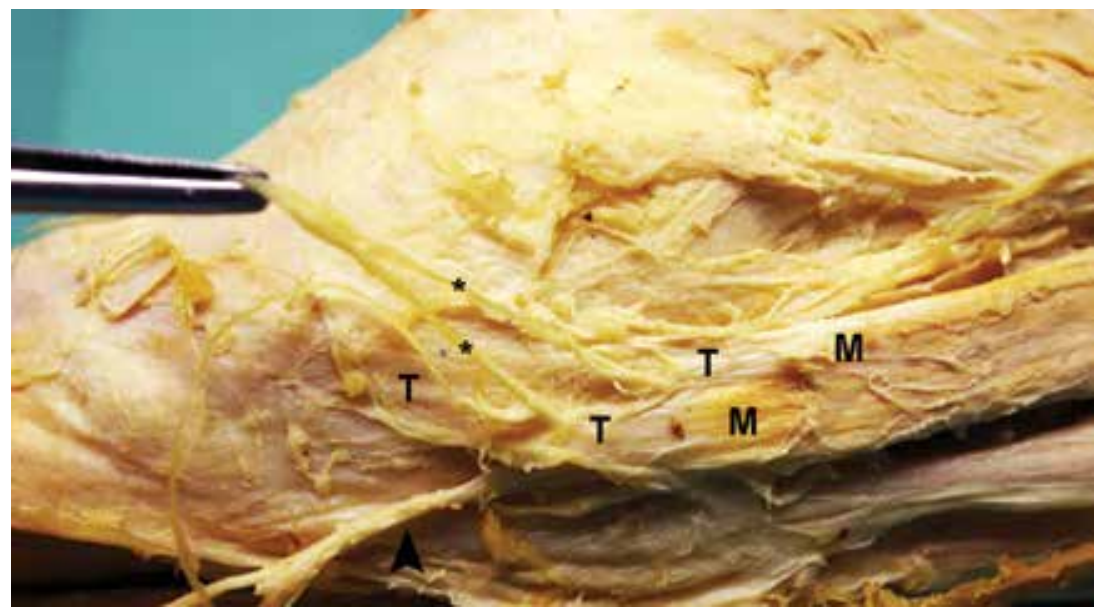

Figure 3. Two infrapatellar branches $\left({ }^{*}\right)$ of the saphenous nerve (arrow heads) passing through the tendon $(\mathrm{T})$ of the Sartorius muscle (M) in the right limb of a male cadaver.

This variation was found unilaterally $(25.9 \%)$ in $3(25 \%)$ male and $4(26.7 \%)$ female cadavers and bilaterally $(7.4 \%)$ in $1(8.3 \%)$ male and $1(6.7 \%)$ female cadaver. In 7 (25.9\%) cases the variation was located on the right side, whereas in $4(14.8 \%)$ cases on the left. In 3 out of 54 limbs (5.6\%), which belonged to a male $(8.3 \%)$ and 2 female $(13.3 \%)$ cadavers, the IPBSN unilaterally penetrated the Sartorius tendon (Figs. 3, 4). In 1 (3.7\%) case the variation was found on the left side and twice on the right (7.4\%). In the remaining $40(74 \%)$ limbs the IPBSN did not run through any fibromuscular hiatus. Results are summarised in Table 1.

\section{DISCUSSION}

At the level of the knee, the IPBSN is susceptible to iatrogenic injury during surgery. Mochida and Kikuchi (1995) [11] reported sensory disturbances of the IPBSN following arthroscopy of the knee joint in $22.2 \%$ of the cases [6]. Patients undergoing anterior cruciate ligament $(A C L)$ reconstruction are at risk of IPBSN injury at a rate of 30-77\% [9]. Moreover, depending on the $A C L$ reconstruction technique, the lateral sural cutaneous nerve and the saphenous nerve may be damaged as well, affecting the rehabilitation programme [2]. Sciatic nerve injury at the hamstrings harvesting site has also been reported [5]. The IPBSN 


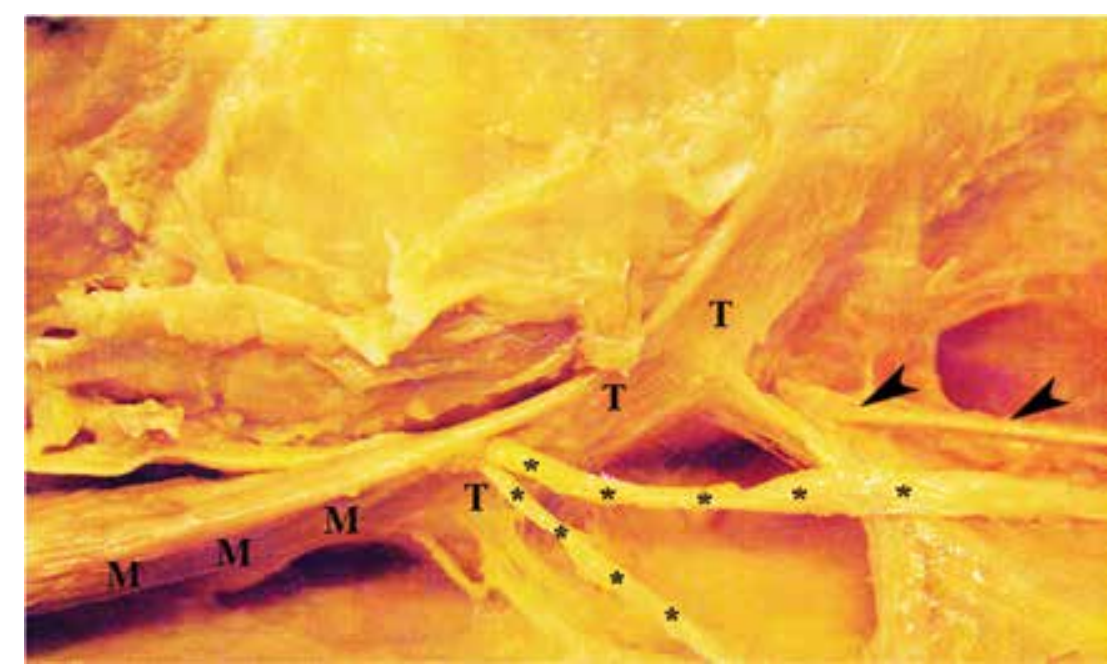

Figure 4. Two infrapatellar branches $\left({ }^{*}\right)$ of the saphenous nerve (arrow heads) passing through the tendon (T) of the Sartorius muscle (M) in the left limb of a female cadaver. Both branches have been detached from the infrapatellar region.

Table 1. Course of the infrapatellar branch of the saphenous nerve in relation to Sartorius muscle in the 54 limbs that were examined in the present study

\begin{tabular}{lc}
\hline Transmuscular & $11(20.4 \%)$ \\
Transtendinous & $3(5.6 \%)$ \\
Extramuscular & $40(74 \%)$ \\
\hline
\end{tabular}

may also be injured by incisions for knee arthrotomies and during tibial nailing [7].

Less than $1 \%$ of adults presenting with lower extremity pain suffer from saphenous neuropathy $[12$, 21]. Although it is not common, differential diagnosis is very difficult and saphenous nerve entrapment neuropathy may be associated with or mimic lumbar radiculopathy (L4 root) [1, 17], patellofemoral disorders [17], suprapatellar plica [17], tear of medial meniscus [13], tibial stress fracture [18], pes anserinus tendinopathy or bursitis $[1,13]$, osteochondritis dissecans [17], nonspecific synovitis [17], and reflex sympathetic dystrophy [19]. The IPBSN entrapment may be caused by mechanical compression of the nerve between the prominent edge of the medial femoral condyle and the tendon of the Sartorius muscle as well as due to the compression of the nerve's course through the Sartorius $[8,15,21]$. This observation has led to several studies on the topographic relationship between the nerve and the Sartorius muscle $[4,11,16]$.

Arthornthurasook and Gaew-Im [2] following the examination of 20 cadavers, classified the anatomical variations of the relation of the IPBSN to the Sartorius muscle into posterior $(62.2 \%)$, penetrating $(21.6 \%)$, parallel (13.5\%) and anterior (2.7\%) types. However, it seems that the parallel type was not accepted by relevant subsequent studies. Specifically, Le Corrollier et al. [10] using ultrasonography in 10 healthy volunteers noticed only two types of IPBSN, the IPBSN posterior to sartorius (in 30\%) and the IPBSN penetrating sartorius (in 70\%). In a recent study, Kalthur et al. [9] dissected 32 male cadavers and observed that in $68.7 \%$ the IPBSN was anterior, in $28.1 \%$ penetrating and in $3.1 \%$ posterior to Sartorius muscle. In another study, the penetrating type presented a very high incidence up to $73 \%[20,21]$.

The present study focused on the penetrating type of IPBSN and aimed to differentiate those nerves that passed through the tendon from those running through the belly of the Sartorius muscle. Thus, presartorial and retrosartorial types of IPBSN were not recorded from the beginning of the study. We distinguished these two situations because we believe that the course of the IPBSN through a tendinous tunnel is more likely to cause mechanical irritation of the nerve and subsequent symptoms of entrapment neuropathy. Indeed, the study by House and Ahmed [7] confirmed this theory, since they performed an open surgical release in two cases of entrapment neuropathy in patients having the penetrating type of IPBSN and in both of them the IPBSN ran through the Sartorius tendon. Furthermore, the same authors pointed out the importance "of the relatively high frequency with which the IPBSN passes through a perforation of hiatus in the Sartorius tendon". In 
Table 2. Modified classification of the infrapatellar branch of the saphenous nerve course in relation to Sartorius muscle

\begin{tabular}{ll}
\hline Type I & Presartorial \\
Type Ila & Muscle-penetrating \\
Type Illb & Tendon-penetrating \\
Type III & Retrosartorial \\
\hline
\end{tabular}

our study, 3 (5.6\%) limbs presented with a tendon-penetrating type of IPBSN. None of 27 cadavers had any known clinical manifestation of entrapment neuropathy in the anatomical corresponding region in their medical history.

The IPBSN is often divided into two or more branches, which is a common finding in sensory nerves. Kalthur et al. [9] observed a single IPBSN in $40.6 \%$, a double IPBSN in $34.3 \%$ and a triple IPBSN in $25 \%$ of their cases. In the study by Veerasethsiri and Tanavalee [21], a single nerve was found in 20\%, double-branched in $67 \%$, and triple-branched in $13 \%$. In the present study 6 limbs with a muscle-penetrating and 2 limbs with a tendon-penetrating type of IPBSN presented a double nerve (57.1\%).

\section{CONCLUSIONS}

The penetrating type of IPBSN includes two distinct anatomical subtypes: a) the muscle-penetrating type and b) the tendon-penetrating type. These two subtypes may also be distinct from a clinical point of view, since the tendon-penetrating type has been associated with IPBSN entrapment neuropathy. In accordance to these findings, we suggest modification of the corresponding classification, as shown in Table 2. Additional and larger clinical studies are necessary to demonstrate if the tendon-penetrating type should be considered as a predisposing factor for IPBSN entrapment neuropathy. Distinguishing the two subtypes might be helpful for that purpose.

\section{REFERENCES}

1. Ahadi T, Raissi GR, Togha M, Nejati P (2010) Saphenous neuropathy in a patient with low back pain. J Brachial PlexPeripher Nerve Inj, 5: 2.

2. Arthornthurasook A, Gaew-Im K (1988) Study of the infrapatellar nerve. Am J Sports Med, 16: 57-59.

3. Committee on Complications of Arthroscopy Association of North America (1985) Complications of arthroscopy and arthroscopic surgery: results of a national survey. Arthroscopy, 1: 214-203.
4. Ebraheim, Nabil A, Mekhail, Anis O (1997) The infrapatellar branch of the saphenous nerve: an anatomic study. J Orthop Trauma, 11: 195-199.

5. Espejo-Baena A, Golano P, Meschian S, Garcia-Herrera JM, Serrano Jimenez JM (2007) Complications in medial meniscus suture: a cadaveric study. Knee Surg Sports Traumatol Arthrosc, 15: 811-186.

6. Figueroa D, Calvo R, Vaisman A, Campero M, Moraga C (2008) Injury to the infrapatellar branch of the saphenous nerve in $\mathrm{ACL}$ reconstruction with the hamstrings technique: clinical and electrophysiological study. Knee, 15: 360-363.

7. House JH, Ahmed K (1977) Entrapment neuropathy of the infrapatellar branch of the saphenous nerve. Am J Sports Med, 5: 217-224.

8. Jameson S, Emmerson K (2007) Altered sensation over the lower leg following hamstring graft anterior cruciate ligament reconstruction with transverse femoral fixation. Knee, 14: 314-320.

9. Kalthur SG, Sumalatha S, Nair N, Pandey AK, Sequeria S, Shobha L (2015) Anatomic study of infrapatellar branch of saphenous nerve in male cadavers. Ir J Med Sci, 184: 201-206.

10. Le Corroller T, Lagier A, Pirro N, Champsaur P (2011) Anatomical study of the infrapatellar branch of the saphenous nerve using ultrasonography. Muscle Nerve, 44: 50-54.

11. Mochida H, Kikuchi S (1995) Injury to infrapatellar branch of saphenous nerve in arthroscopic knee surgery. Clin Orthop Relat Res, 320: 88-94.

12. Mumenthaler M, Schlaick H (1991) Peripheral nerve lesions, diagnosis and therapy. Thieme Medical Publishers, New York.

13. Noble J, llango B, Obeid M (1998) Complications of arthroscopy of the knee. Knee, 5: 1-8.

14. Papastergiou SG, Voulgaropoulos $H$, Mikalef $P$, Ziogas $E$, Pappis G, Giannakopoulos I (2006) Injuries to the infrapatellar branch(es) of the saphenous nerve in anterior cruciate ligament reconstruction with four-strand hamstring tendon autograft: vertical versus horizontal incision for harvest. Knee Surg Sports Traumatol Arthrosc, 14: 789-793.

15. Peck E, Finnoff JT, Smith J (2010) Neuropathies in runners. Clin Sports Med, 29: 437-457.

16. Pendergrass TL, Moore JH (2004) Saphenous neuropathy following medial knee trauma. J Orthop Sports Phys Ther, 34: 328-324.

17. Poehling GG, Pollock FE Jr, Koman LA (1988) Reflex sympathetic dystrophy of the knee after sensory nerve injury. Arthroscopy, 4: 31-35.

18. Porr J, Chrobak K, Muir B (2013) Entrapment of the saphenous nerve at the adductor canal affecting the infrapatellar branch: a report on two cases. J Can Chiropr Assoc, 57: 341-349.

19. Saal JA, Dillingham MF, Gamburd RS, Fanton GS (1988) The pseudoradicular syndrome. Lower extremity peripheral nerve entrapment masquerading as lumbar radiculopathy. Spine (Phila Pa 1976), 13: 926-930.

20. Vardi G (2004) Sciatic nerve injury following hamstring harvest. Knee, 11: 37-39.

21. Veerasethsiri P, Tanavalee A (2014) Infrapatellar branch of the saphenous nerve: a cadaveric study. Thai J Orthopaedic Surg, 38: 17-20. 